\title{
Richesse des problèmes posés et créativité des solutions soumises dans la Communauté d'apprentissages scientifiques et mathématiques interactifs (CASMI)
}

\author{
Dominic Manuel \\ Viktor Freiman \\ Jimmy Bourque \\ Université de Moncton
}

\section{Résumé}

Cette étude s'intéresse à la richesse des problèmes mathématiques posés et à la créativité des solutions soumises par les membres de la Communauté d'apprentissages scientifiques et mathématiques interactifs (CASMI), une ressource virtuelle destinée aux élèves francophones du Nouveau-Brunswick et d'ailleurs. L'exploration des problématiques identifiées par les chercheurs préoccupés par les rares occasions qu'ont les élèves de résoudre des problèmes riches et de développer leur créativité en classe nous amène à construire un cadre conceptuel afin 1) d'analyser la richesse des problèmes proposés dans la CASMI, 2) d'évaluer la créativité des solutions soumises par les membres de cette communauté virtuelle et 3 ) de déterminer s'il existe une relation entre la richesse des problèmes posés et la créativité des solutions soumises. Les résultats révèlent que les problèmes plus riches semblent susciter plus de solutions originales et des réponses divergentes. Cependant, ces résultats mettent aussi en évidence le besoin d'élargir le cadre conceptuel sous-jacent à la formulation des problèmes mathématiques riches offerts aux élèves et de mener des recherches plus approfondies dans ce domaine.

\section{Abstract}

This research focuses on the richness of mathematical problems posted and the creativity of the solutions submitted by members of the CASMI (Communauté d'apprentissages scientifiques et mathématiques interactifs), a virtual resource used by Francophone students from New Brunswick and elsewhere. After reviewing issues identified by researchers preoccupied by the few opportunities offered to students to solve rich mathematical problems and develop their creativity in the classroom, we develop a conceptual framework in order to : 1) analyze the richness of the mathematical problems posted on the CASMI website; 2) assess the creativity of the solutions to the problems submitted on this website; and 3) verify the link between the richness of the problems and the mathematical creativity of the solutions. Our results suggest that rich mathematical problems bring more original solutions and multiple answers. These results also reveal the need for a broader conceptual framework in order to enhance the richness of mathematical problems offered to students, as well as for continuing research in this area. 


\section{Introduction}

Au XXle siècle, les citoyennes et les citoyens doivent être capables d'aborder des problèmes complexes, qui ne se résolvent pas toujours par une simple application d'une stratégie ou d'un algorithme quelconque. La résolution de ces problèmes requiert plutôt la mobilisation d'habiletés de haut niveau cognitif pour pouvoir construire une démarche qui fait preuve de créativité (Sheffield, 2008). Les élèves francophones du Nouveau-Brunswick et d'ailleurs au Canada ont-ils les occasions nécessaires pour développer les habiletés permettant d'aborder ce type de problèmes? Ayant pour mission de « favoriser le développement de personnes autonomes, créatrices et épanouies », le Ministère de l'Éducation et du Développement de la Petite Enfance du Nouveau-Brunswick (MÉDPENB, 2011, p. 5) met l'accent sur le développement de la pensée critique et créative parmi les résultats d'apprentissages transdisciplinaires que les élèves doivent atteindre et ce, dans toutes les matières scolaires, incluant les mathématiques. Cependant, les résultats des évaluations en mathématiques de l'étude PISA en 2009 (Knighton, Brochu et Gluszynski, 2010) semblent indiquer que ces élèves pourraient profiter d'une offre plus fréquente d'occasions de développer leur créativité mathématique. En effet, si les jeunes francophones de 15 ans ont des résultats inférieurs à leurs collègues anglophones partout au Canada, les jeunes du Nouveau-Brunswick, en particulier, se situent à l'avant dernier rang parmi ceux des provinces canadiennes. Ces résultats pourraient s'expliquer par un manque de ressources disponibles. Un tel manque a été bien documenté par plusieurs chercheurs, dont Poirier (2010), comme un facteur ayant un impact sur les apprentissages chez les élèves vivant dans un milieu minoritaire francophone. De là l'intérêt d'analyser la créativité dans les problèmes posés dans la Communauté d'apprentissages scientifiques et mathématiques interactifs (CASMI), puisque cette communauté virtuelle est l'une des rares ressources conçues dans et pour un milieu minoritaire francophone.

\section{Contexte de l'étude}

Au cœur des orientations prônées par le National Council of Teachers of Mathematics (NCTM) à l'endroit de l'enseignement et de l'apprentissage des mathématiques, on retrouve la résolution de problèmes comme élément primordial pour les élèves (NCTM, 2000). Le concept même de résolution de problèmes a évolué en mettant plus d'accent sur les habiletés à résoudre des problèmes complexes dans un contexte signifiant. Ainsi, le concept didactique de situation-problème a été introduit comme alternative à la résolution de problèmes d'application où les données sont bien définies et les procédures de résolution ainsi que le résultat attendu sont connus avant même de 
les résoudre (Pallascio, 2005). Placé devant une situation-problème, l'élève est engagé dans des activités de production et non de reproduction, ce qui l'amène à : 1) employer et parfois concevoir différentes stratégies de compréhension, de résolution, d'organisation et de communication; 2) formuler des hypothèses et des conjectures; 3 ) intérioriser les « règles du jeu »; 4) développer les habiletés à chercher, créer, intuitionner, analyser, synthétiser, justifier et anticiper le résultat et 5) mobiliser et utiliser de façon efficace les ressources pour élaborer une ou plusieurs réponses (Corbeil, Pelletier et Pallascio, 2001). De plus, nombreux sont les auteurs qui voient les mathématiques comme une discipline ayant le potentiel de développer la créativité et soulignent l'importance de renforcer celle-ci en salle de classe (Petrowski, 2000; Robinson, 2009; Sriraman, 2004). Ces diverses habiletés ne sont pas innées chez les élèves et ne s'actualisent pas automatiquement. Par conséquent, il devient important de les cultiver et de les nourrir (Mann, 2005; Sheffield, 2008).

Par contre, malgré l'importance de prêter attention au développement de la créativité en mathématiques prônée par les chercheurs et les courants réformistes des curricula modernes, le changement réel quant aux pratiques pédagogiques se fait toujours attendre (Lester et Kehle, 2003; Robinson, 2009). Par exemple, Chan (2008) se demande si les approches pédagogiques tiennent compte des besoins de l'élève à s'exprimer et à se développer selon ses talents, son style et son rythme d'apprentissage. Aussi, Hashimoto (1997) et Poirier (2001) questionnent les croyances des enseignants et des élèves, car certains pensent encore qu'il n'y a qu'une seule bonne réponse et qu'une seule façon de résoudre un problème mathématique. De son côté, Mann (2006) avance que les enseignants en salle de classe sont parfois portés à proposer aux élèves des questions et des exercices exigeant des réponses rapides. Pourtant, la créativité se développe par un processus plutôt lent et qui demande du temps de réflexion (Mann, 2005). De plus, les enseignants ont tendance à enseigner aux élèves les stratégies spécifiques pour résoudre les problèmes d'un certain type (Chan, 2008). En procédant ainsi, ils ne donnent pas l'occasion aux élèves d'exercer leurs habiletés de pensée divergente, c'est-à-dire de chercher différentes possibilités de solutions et diverses stratégies possibles pour résoudre les problèmes. Comme conséquence, en proposant des stratégies de résolution de problèmes aux élèves de façon explicite, les enseignants privent ces derniers des occasions de créer leurs propres représentations et stratégies, et de possiblement arriver à des réponses différentes, mais tout aussi correctes (Mann, 2005). Selon Meissner (2005), l'enseignement explicite de stratégies de résolution de problèmes risque de nuire à la curiosité naturelle des apprenants et à leur enthousiasme envers les mathématiques, au fur et à mesure qu'ils avancent dans leur scolarité.

Pour contrer ces problèmes, plusieurs auteurs proposent des stratégies qui auraient le potentiel de développer davantage la créativité mathématique en salle de classe. Cline (1999), Sheffield (2003), Freiman (2006), ainsi que Freiman et Sriraman (2007) affirment que la créativité mathématique se développe lors de la résolution de tâches riches en mathématiques, lesquelles se présentent sous forme de problèmes ouverts. Contrairement aux problèmes fermés ayant seulement une solution, les problèmes ouverts peuvent donner aux élèves une occasion de les résoudre à leur façon et selon leurs habiletés (Klavir et Hershkovitz, 2008). De plus, ces types de problèmes stimulent de plus hauts niveaux cognitifs, et ce, pour plusieurs raisons : ils ouvrent la voie à plusieurs interprétations, stratégies et réponses possibles; ils permettent aux élèves de construire leurs connaissances dans une variété de contextes; les élèves peuvent affronter le même problème sous différentes perspectives, se représenter les concepts 
et les relations mathématiques de diverses façons, jusqu'à ce qu'ils découvrent une stratégie qui leur permettra d'étudier avec rigueur le problème, tout en y apportant leurs propres inventions et en faisant leurs propres découvertes. Dans le contexte d'un problème ouvert, les élèves peuvent prendre des risques pour trouver différentes façons de résoudre un problème, plusieurs possibilités de réponses, ainsi que développer des stratégies originales, ce qui caractérise la créativité mathématique (Hershkovitz, Peled et Littler, 2009; Mann, 2005). Cependant, les chercheurs notent que l'utilisation de problèmes ouverts en salle de classe est un phénomène plutôt rare (Cline, 1999; Freiman, 2006; Freiman et Sriraman, 2007; Sheffield, 2003).

Les communautés virtuelles d'apprentissage sont aussi mentionnées comme solution ayant le potentiel de développer la créativité en mathématiques. Entre autres, Renninger et Shumar (2004) discutent du potentiel créatif du site Math Forum ${ }^{1}$ qui est orienté vers la résolution de problèmes à la fois authentiques et contextualisés, et qui fait appel à une communication et à un raisonnement mathématique propres à l'élève. À leur tour, les chercheurs étudiant le site $N R I \mathrm{CH}^{2}$ expriment leur conviction par rapport au processus créatif dans la résolution de problèmes, processus à la base des mathématiques en tant qu'activité humaine (Piggott, 2007). Cependant, les écrits cités ne précisent pas comment la créativité est définie et mesurée lors de la résolution de problèmes, ce qui semble être important à examiner en profondeur à la lumière des enjeux identifiés dans les paragraphes précédents. Il est à noter que les deux communautés virtuelles mentionnées ici sont des ressources anglophones.

\section{Position du problème et objectifs de la recherche}

La considération des deux solutions qui précèdent, à savoir la résolution de problèmes riches et l'utilisation des communautés virtuelles, nous amène à examiner davantage la Communauté d'apprentissages scientifiques et mathématiques interactifs $(\mathrm{CASMI})^{3}$, une ressource créée par une équipe de chercheurs de l'Université de Moncton et destinée aux écoles francophones de la province et d'ailleurs au Canada, et dans d'autres pays. Cette ressource virtuelle est par ailleurs une des rares qui n'est pas traduite de l'Anglais et qui a été créée par des francophones pour être utilisée dans les écoles francophones du Nouveau-Brunswick. Selon les concepteurs de la CASMI, cette ressource met l'accent sur la résolution de problèmes riches en mathématiques, en sciences et dans les jeux d'échecs (Freiman et Lirette-Pitre, 2009; Freiman, Lirette-Pitre et Manuel, 2007; Freiman, Manuel et Lirette-Pitre, 2007). De plus, selon Freiman et Manuel (2007), des entrevues auprès d'un petit échantillon d'utilisateurs de la CASMI, élèves et enseignants, révèlent que leurs tentatives de trouver des réponses aux problèmes posés a stimulé l'utilisation de stratégies diversifiées pour certains problèmes, ce qui laisse entrevoir une certaine créativité dans la recherche de solutions. Cependant, la richesse des problèmes et son influence sur la créativité des solutions soumises de façon électronique par les utilisateurs de la CASMI n'ont pas été validées de façon empirique. La question à savoir s'il y a réellement des problèmes riches et des solutions créatives dans cet espace virtuel demeure donc jusqu'ici sans réponse.

\footnotetext{
${ }^{1}$ Disponible à l'adresse URL suivante : http://www.mymathforum.com/

${ }^{2}$ Disponible à l'adresse URL suivante : $\underline{\text { http://nrich.maths.org/publicl }}$

${ }^{3}$ Le site porte maintenant le nom CAMl pour Communauté d'apprentissages multidisciplinaires interactifs et est accessible à l'adresse URL suivante : www.umoncton.ca/cami
} 
En analysant de façon systématique le contenu des problèmes mathématiques posés dans la communauté virtuelle, ainsi que la créativité des solutions soumises par les utilisateurs, nous serons en mesure de déterminer si ces problèmes favorisent la créativité mathématique. Nos objectifs de recherche sont alors : 1) analyser la richesse des problèmes proposés dans la CASMl; 2) évaluer la créativité mathématique des solutions soumises par les utilisateurs de la CASMI aux différents problèmes posés et 3 ) déterminer s'il existe une relation entre la richesse des problèmes mathématiques posés dans la CASMI et la créativité mathématique des solutions soumises. Mais à quelles réalités renvoient les notions de richesse des problèmes mathématiques et de créativité mathématique?

\section{Cadre conceptuel de la recherche}

Pour définir le problème mathématique riche, nous avons retenu des critères pouvant être évalués dans les énoncés des problèmes. Selon différents auteurs, un problème est riche lorsqu'il est ouvert (Diezmann et Watters, 2004; Takahashi, 2000), complexe (Diezmann et Watters, 2004; Schleicher, 1999), mal défini (Murphy, 2004), contextualisé (Greenes, 1997) et possède diverses interprétations possibles (Hancock, 1995). Plus précisément, le problème est ouvert s'il contient plusieurs réponses correctes possibles ou peut se résoudre en utilisant plusieurs stratégies (Takahashi, 2000). Le problème complexe respecte le plus de critères possibles parmi les suivants : il se résout en utilisant plus d'une étape; il demande implicitement ou explicitement de trouver des régularités, de généraliser des résultats ou de faire des preuves mathématiques; il demande explicitement de faire des choix et de les justifier, et il demande explicitement de poser d'autres problèmes afin de continuer l'exploration. Un problème est mal défini s'il lui manque certaines données importantes ou nécessaires, mais qui peuvent être définies ou recherchées, s'il inclut des données superflues ou encore, s'il ne fournit pas les informations suffisantes pour pouvoir le résoudre (aucune solution ou problème impossible) (Kitchner, 1983). Le problème à diverses interprétations est un problème suscitant différentes visions possibles du problème menant à différentes réponses possibles. Enfin, le problème contextualisé est celui qui se présente dans des situations réelles tirées de la vie sociale d'un jeune ou d'un adulte ou dans des situations fictives.

Le schéma conceptuel présenté à la figure 1 représente la vision du problème mathématique riche adoptée pour cette étude. Dans ce schéma, les rectangles représentent les cinq caractéristiques retenues dans la définition du problème mathématique riche, tandis que les bulles correspondent aux critères observables dans les énoncés des problèmes écrits. Notons que les caractéristiques et les critères adoptés pour évaluer la richesse des problèmes constituent des choix : d'autres chercheurs auraient pu privilégier une approche différente. Notre choix porte sur des critères déjà reconnus dans les écrits scientifiques du domaine et qui peuvent s'interpréter de façon suffisamment univoque pour assurer la cohérence de l'analyse quantitative.

En ce qui a trait à la créativité, définir celle-ci constitue une problématique en soi. En effet, Treffinger, Young, Selby et Shepardson (2002) mentionnent que les chercheurs ne s'entendent pas sur une seule et unique définition de la créativité. Selon Mann (2005), nous pouvons compter au-delà de 100 définitions et cette vaste quantité de définitions est une des raisons pour lesquelles très peu de recherches ont été entreprises sur le sujet. 


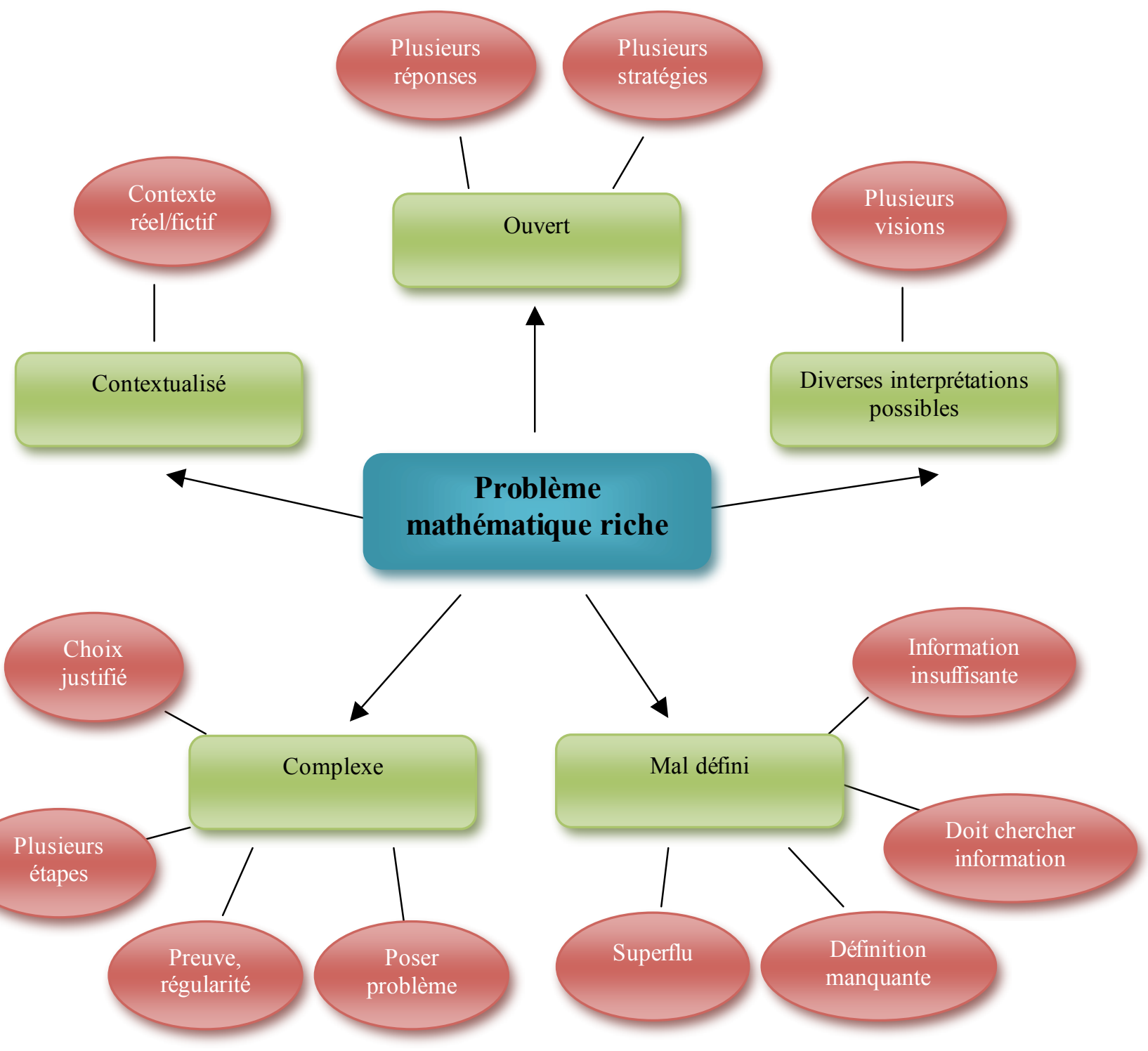

Figure 1: Schéma conceptuel du problème riche

Entre autres, Runco (1993) définit la créativité comme étant un modèle complexe qui met l'accent sur les caractéristiques personnelles suivantes : les pensées convergente et divergente dans la résolution de problèmes; l'habileté à créer et à rechercher des problèmes; la façon de s'exprimer; la motivation intrinsèque; l'aptitude à se questionner et une bonne estime de soi. Haylock (1987) conclut que la créativité est plutôt la capacité de déterminer de nouvelles relations entre des idées et des applications, de faire de nouvelles associations entre des idées n'ayant pas été liées auparavant. De son côté, Krutetskii (1976) caractérise la créativité mathématique comme étant l'habileté à poser un problème ainsi que l'inventivité et l'originalité des solutions. 
Mann (2005), Haylock (1997) et Singh (1988) retiennent la fluence, la flexibilité et l'originalité des solutions correctes à un problème mathématique comme caractéristiques clés de la créativité mathématique. Ce sont ces composantes que nous avons retenues pour notre définition de la créativité mathématique. La fluence renvoie au nombre de réponses correctes ou au nombre de problèmes créés dans un espace virtuel collectif de solutions, tandis que la flexibilité représente le nombre de stratégies efficaces différentes dans ce même regroupement de solutions. La distinction entre fluence et flexibilité tient compte du fait qu'une même stratégie peut être utilisée pour arriver à plusieurs réponses différentes ou que plusieurs stratégies distinctes peuvent être utilisées pour arriver à une même réponse. Pour sa part, l'originalité se réfère aux réponses et aux stratégies correctes peu fréquentes dans un espace virtuel collectif. Un tel espace virtuel collectif de solutions renvoie à l'ensemble des solutions à un problème trouvées par des utilisateurs ou membres, et soumises de façon électronique dans une communauté virtuelle d'apprentissage. Cette définition s'inspire de la définition d'espace collectif de solution énoncée par Leikin (2007).

\section{Cadre méthodologique et outils de collecte de données}

Cette recherche quantitative se veut une recherche nomothétique exploratoire, selon la typologie de Van der Maren (1996). II s'agit d'une analyse du contenu des problèmes posés dans le site Web de la CASMI et des solutions trouvées et soumises de façon électronique par les membres de cette communauté virtuelle. En 2009, au moment de l'étude, la communauté virtuelle compte 28341 membres, dont $88 \%$ sont des élèves. Ces membres proviennent de 40 pays différents. Plus de la moitié des membres sont Canadiens et $83 \%$ de ceux-ci sont du Nouveau-Brunswick. Selon la rubrique "Statistiques » située dans l'interface administrative du site de la CASMI, ce sont presque toujours les membres du Nouveau-Brunswick qui visitent le site.

La démarche de recherche utilisée se divise en trois étapes, chacune portant sur un des objectifs. En premier lieu, la richesse des 180 problèmes posés dans le site de la CASMI a été analysée en utilisant une grille créée et validée à cette fin. Au départ, cette grille définissait d'une manière opérationnelle les 12 critères retenus pour chacune des 5 caractéristiques des problèmes riches faisant partie de notre schéma conceptuel. Cette grille originale a fait l'objet d'une validation inter-codeurs. Cinq codeurs ont évalué la richesse de 10 problèmes choisis au hasard à l'aide de la grille. À la suite de l'examen des pourcentages d'accord et d'une discussion avec les codeurs, deux critères ayant des pourcentages d'accords inférieurs à $80 \%$ ont été éliminés, l'un portant sur les problèmes à diverses interprétations, l'autre sur les problèmes mal définis incluant des données superflues. De plus, les codeurs ont suggéré de combiner en un seul les deux critères traitant des données manquantes dans la catégorie du problème mal défini, afin d'éviter des confusions. Enfin, malgré son intérêt, un critère de la catégorie du problème ouvert portant sur les demandes explicites de trouver plusieurs solutions n'a pas été considéré puisque les problèmes possédant cette particularité se trouvaient déjà inclus dans le critère portant sur les problèmes à plusieurs réponses correctes possibles. Ainsi, la grille d'analyse de la richesse des problèmes utilisée dans cette étude porte sur les 8 critères retenus à la suite du processus de validation. Chacun des 180 problèmes analysés a reçu un point par critère respecté, pour un score de richesse total possible de 8 points (voir annexe A).

En deuxième lieu, pour évaluer la créativité mathématique des espaces virtuels collectifs de solutions, 50 problèmes ont été choisis de façon aléatoire. Ces problèmes contenaient entre 75 et 402 solutions soit, en moyenne, 
187 solutions par problème. Les solutions ont été analysées en utilisant, encore une fois, une grille créée et validée à cette fin. Cette grille contient les règles suivies pour attribuer les scores pour les trois composantes de la créativité mathématique retenues, c'est-à-dire la fluence, la flexibilité et l'originalité (voir annexe B). Avant d'être utilisée pour évaluer la créativité des solutions, cette grille a, elle aussi, subi une validation inter-codeurs. Deux codeurs ont appliqué la grille d'analyse à 100 solutions soumises à un des problèmes choisi au hasard. Étant donné le pourcentage d'accord élevé, la grille n'a pas été modifiée.

En troisième lieu, pour vérifier l'existence d'une relation entre la richesse des problèmes posés dans la CASMI et la créativité mathématique des solutions, nous avons utilisé le test du rapport de vraisemblance (khi-carré) (Field, 2005). Ce test a été choisi après avoir effectué une analyse préliminaire des données (Tabachnick et Fidell, 2007) qui a révélé que les distributions des scores pour les variables « fluence » et « originalité » n'étaient pas normales. Les scores $Z$ de la voussure et de l'asymétrie pour ces deux variables étaient supérieurs en valeur absolue à la valeur critère de 3,29. Cette constatation a fait en sorte qu'aux fins de comparaisons, nous avons transformé tous les scores pour toutes nos variables en catégories, telles que présentées au tableau 1. Pour la richesse des problèmes, trois catégories ont été créées: 1) les problèmes peu riches, ayant un score de 3 ou moins, 2) les problèmes moyennement riches, ayant un score de 4, et 3 ) les problèmes riches, ayant un score de 5 et plus. Ces catégories ont été déterminées en fonction de la fréquence relative des scores, les scores peu fréquents étant regroupés dans une même catégorie. Pour la créativité, les composantes de fluence et d'originalité comprennent chacune deux catégories (une bonne réponse ou plusieurs bonnes réponses; solutions originales ou non originales), alors que la flexibilité compte 4 catégories en fonction du nombre de stratégies utilisées dans la solution au problème. Ce sont les fréquences relatives des problèmes et solutions classés dans ces diverses catégories qui ont été soumises aux analyses statistiques.

Tableau 1

Catégories de variables formées à la suite des tests statistiques préliminaires

\begin{tabular}{|c|c|c|}
\hline \multicolumn{3}{|c|}{ RICHESSE DU PROBLĖME MATHÉMATIQUE } \\
\hline Variable & Scores & Nom de la catégorie formée \\
\hline \multirow{3}{*}{$\begin{array}{l}\text { Richesse du } \\
\text { problème } \\
(\mathrm{N}=180)\end{array}$} & 1 à 3 & Problèmes peu riches \\
\hline & 4 & Problèmes moyennement riches \\
\hline & 5 à 8 & Problèmes riches \\
\hline \multicolumn{3}{|r|}{ CRÉATIVITÉ MATHÉMATIQUE } \\
\hline Variable & Scores & Nom de la catégorie formée \\
\hline \multirow{2}{*}{$\begin{array}{l}\text { Fluence } \\
(\mathrm{N}=50)\end{array}$} & 1 & Une bonne réponse \\
\hline & 2 ou plus & Plusieurs bonnes réponses \\
\hline \multirow{4}{*}{$\begin{array}{l}\text { Flexibilité } \\
(N=50)\end{array}$} & 1 & 1 stratégie \\
\hline & 2 & 2 stratégies \\
\hline & 3 & 3 stratégies \\
\hline & 4 ou plus & Plus de 3 stratégies \\
\hline \multirow{2}{*}{$\begin{array}{c}\text { Originalité } \\
(N=50)\end{array}$} & 0 & Solutions non originales \\
\hline & Autres & Solutions originales \\
\hline
\end{tabular}




\section{Analyse des résultats}

Les résultats sont présentés et analysés ci-après en ce qui a trait à la richesse des problèmes posés dans la CASMI, à la créativité dans les espaces virtuels de solutions et à l'existence d'un lien entre la richesse des problèmes mathématiques et la créativité des solutions soumises.

\section{La richesse des problèmes posés dans la CASMI}

En utilisant les tests de normalité, il ressort que les scores de richesse des 180 problèmes posés dans le site CASMI et analysés dans cette étude sont presque normalement distribués (score $Z$ de l'asymétrie de 0,546 et score Z de la voussure de 0,489). La moyenne des scores se situe à 4 , sur une possibilité maximale de 8 , avec un écart-type de 1,27. D'emblée, ces chiffres indiquent que la richesse des problèmes posés dans la CASMI s'avère plutôt modeste.

En poursuivant l'analyse des fréquences relatives des scores obtenus pour chacun des critères retenus dans la grille d'évaluation de la richesse des problèmes (voir tableau 2), il appert que quatre critères sont respectés dans la grande majorité des problèmes: plusieurs réponses correctes, 61,1\%; plusieurs stratégies efficaces possibles, 94,4\%; problèmes se résolvant en utilisant plusieurs étapes, 87,8\%; et problèmes contextualisés, 90,6 \%. Par contre, les quatre autres critères sont plutôt négligés : problèmes demandant de faire des choix et de les justifier, 11,7 \%; problèmes menant à la création et à l'étude d'autres questions, 2,8 \%; problèmes menant à la découverte des régularités et à la généralisation des résultats, 28,9\%; et problèmes mal définis à cause d'un manque de données ou d'information, $22,8 \%$. Cette répartition des fréquences en fonction des critères indique une vision relativement restreinte du concept de créativité reflétée dans les problèmes posés dans la CASMI.

Tableau 2

Fréquences relatives des scores obtenus pour les critères de richesse retrouvées dans les 180 problèmes posés dans la CASMI

\begin{tabular}{|c|c|}
\hline \multicolumn{1}{|c|}{ Liste des critères } & $\%$ \\
\hline CATÉGORIE : PROBLĖME OUVERT & 61,1 \\
\hline Plusieurs réponses correctes possibles & 94,4 \\
\hline Plusieurs stratégies possibles pour résoudre le problème & 87,8 \\
\hline CATÉGORIE : PROBLĖME COMPLEXE & 11,7 \\
\hline Plusieurs étapes & 2.8 \\
\hline Demande explicite de justifier les choix & 28,9 \\
\hline Demande explicite de poser d'autres questions, problèmes & \\
\hline Découverte de régularités, généralisations ou preuves & 22,8 \\
\hline CATÉGORIE : PROBLĖME MAL DÉFINI & \\
\hline Manque de données ou informations nécessaires pour résoudre le problème & 90,8 \\
\hline CATÉGORIE : PROBLĖME CONTEXTUALISÉ
\end{tabular}


En somme, les problèmes posés dans la CASMI s'avèrent relativement modestes en termes de richesse mathématique, et cette richesse repose en grande partie sur quatre critères de base. II s'agit essentiellement de problèmes ouverts ayant plusieurs réponses possibles ou permettant l'utilisation de plusieurs stratégies efficaces pour les résoudre, de problèmes complexes à plusieurs étapes et de problèmes contextualisés.

\section{La créativité dans les espaces virtuels de solutions des problèmes de la CASMI}

L'analyse des composantes de la créativité dans les espaces virtuels collectifs de solutions montre globalement que la très grande majorité des répondants se limite à une seule réponse et une seule stratégie de résolution de problèmes, et ces dernières sont souvent peu originales. Toutefois, nous avons pu quand même observer des traces de créativité mathématique dans les espaces virtuels collectifs de solutions, comme le montrent les résultats suivants en fonction des trois dimensions de la créativité retenues.

Pour la fluence, nous avons observé deux réponses correctes ou plus soumises dans les espaces virtuels collectifs de solutions pour $48 \%$ des problèmes, tandis que $52 \%$ des espaces de solutions contenaient seulement une seule réponse correcte. Comme présenté au tableau 3, la fréquence relative des problèmes qui ont suscité deux réponses correctes se situe à $12 \%$. La fréquence des problèmes suscitant plus de deux réponses correctes sans demande explicite s'élève à $30 \%$, et avec demande explicite, à $6 \%$. Ces chiffres indiquent qu'environ la moitié des problèmes posés dans la CASMI suscitent plus d'une réponse correcte et que ces réponses sont en majorité trouvées sans demande explicite.

Tableau 3

Fréquences relatives des catégories composant la variable de créativité mathématique

\begin{tabular}{|c|c|c|c|}
\hline \multirow{6}{*}{ Fluence } & \multicolumn{2}{|r|}{ Catégories } & \multirow{2}{*}{$\begin{array}{c}\text { Fréquence relative en } \% \\
52\end{array}$} \\
\hline & \multicolumn{2}{|c|}{1 réponse correcte } & \\
\hline & \multirow{4}{*}{$\begin{array}{c}\text { Plus d'une } \\
\text { réponse } \\
\text { correcte }\end{array}$} & 2 réponses correctes & 12 \\
\hline & & Plus de 2 (sans demande explicite) & 30 \\
\hline & & Plus de 2 (avec demande explicite) & 6 \\
\hline & & Total & 48 \\
\hline \multirow{4}{*}{ Flexibilité } & \multicolumn{2}{|l|}{1 stratégie } & 24 \\
\hline & \multicolumn{2}{|l|}{2 stratégies } & 36 \\
\hline & \multicolumn{2}{|l|}{3 stratégies } & 26 \\
\hline & \multicolumn{2}{|c|}{4 stratégies ou plus } & 14 \\
\hline \multirow{2}{*}{ Originalité } & \multicolumn{2}{|c|}{ Problèmes contenant des solutions originales } & 44 \\
\hline & \multicolumn{2}{|c|}{ Solutions non originales } & 56 \\
\hline
\end{tabular}

Pour ce qui est de la flexibilité (voir tableau 3), nous avons observé une variation relativement importante dans les stratégies utilisées. Seulement $24 \%$ des problèmes sont résolus en utilisant une stratégie. Par contre, dans $36 \%$ des problèmes, deux stratégies différentes sont utilisées. Dans $24 \%$ des problèmes, on trouve trois stratégies différentes et pour le reste des problèmes (14\%), on en trouve quatre ou plus. Nous avons quand même remarqué que quelques stratégies (par exemple : procéder par tâtonnement) étaient très souvent utilisées. 
Finalement, pour l'originalité (voir tableau 3), des solutions originales se retrouvent dans $44 \%$ des problèmes analysés, tandis que dans $56 \%$ des problèmes, les mêmes réponses correctes et des stratégies semblables sont utilisées. Le fait que plusieurs solutions analysées étaient semblables en termes de contenu nous a amené à faire l'hypothèse que les répondants travaillaient en groupe pour résoudre les problèmes et que chacun, par la suite, avait soumis la solution utilisée par le groupe dans l'espace virtuel collectif de solutions, ce qui a probablement eu une influence sur le plan de l'originalité des solutions.

Ainsi, en termes de créativité, ces résultats montrent que celle-ci se révèle dans un peu moins de la moitié des solutions soumises par les participants dans les espaces virtuels collectifs. Des trois composantes de la créativité, la flexibilité apparaît comme la plus importante, se laissant entrevoir dans les trois-quarts des problèmes, alors que la fluence et l'originalité ont été notées dans un peu moins de la moitié des problèmes.

\section{Relation entre la richesse des problèmes et la créativité}

En réponse à notre objectif concernant l'existence d'une relation entre la richesse des problèmes posés sur le site de la CASMI et la créativité mathématique des espaces virtuels collectifs de solutions aux problèmes, les résultats du test du rapport de vraisemblance (khi-carré) (voir tableau 4) révèlent un lien de dépendance statistiquement significatif $\left(L^{2}[2]=9,706, p=0,008\right)$ entre la fluence et la richesse du problème mathématique. II existe aussi un lien de dépendance statistiquement significatif $\left(L^{2}[2]=10,07, p=0,007\right)$ entre l'originalité et la richesse du problème mathématique. La taille de l'effet entre ces variables, déterminée à l'aide du V de Cramer, est moyenne dans les deux cas, se situant respectivement à 0,422 et 0,441. Par contre, il n'y a pas de lien de dépendance statistiquement significatif $\left(L^{2}[2]=7,718, p=0,260\right)$ entre la flexibilité et la richesse du problème mathématique. Ce dernier résultat s'explique peut-être par le fait que le critère consistant à susciter plusieurs stratégies de résolution de problème est celui qui se rencontre le plus souvent parmi les critères de richesse mathématique et ce, dans presque $95 \%$ des problèmes.

Tableau 4

Résultats du test du rapport de vraisemblance entre la richesse du problème et les variables de la créativité $(\mathrm{N}=50)$.

\begin{tabular}{|l|c|c|c|c|}
\hline $\begin{array}{c}\text { Relation étudiée entre la } \\
\text { richesse du problème et }\end{array}$ & $\begin{array}{c}\text { Rapport de } \\
\text { vraisemblance } L^{2}\end{array}$ & dl & Sign. & V de Cramer \\
\hline la fluence & 9,706 & 2 & 0,008 & 0,422 \\
\hline la flexibilité & 7,718 & 6 & 0,260 & 0,268 \\
\hline ''originalité & 10,07 & 2 & 0,007 & 0,441 \\
\hline
\end{tabular}

\section{Discussion}

Les résultats de cette étude montrent que parmi les huit attributs utilisés pour évaluer la richesse mathématique des problèmes posés dans la CASMI, quatre sont fréquemment rencontrés : le problème qui possède plusieurs bonnes réponses possibles, qui peut se résoudre en appliquant plusieurs stratégies différentes, qui se résout 
en plusieurs étapes et qui est contextualisé. Ces résultats semblent confirmer l'intention initiale de bâtir le site de résolution de problèmes complexes, signifiants et contextualisés permettant aux élèves de développer une démarche, ainsi que raisonner et communiquer mathématiquement, conformément aux principes didactiques énoncés dans le programme d'études au Nouveau-Brunswick (Langlais, Vézina et Freiman, 2005). Ces principes demandent explicitement de proposer aux élèves une situation-problème complexe, signifiante et contextualisée afin qu'ils puissent la gérer en construisant une démarche de résolution mathématiquement valide et bien justifiée (raisonnement mathématique), communiquée de façon claire et mathématiquement correcte. De plus, ce processus doit amener l'élève à développer la capacité de faire des liens entre les mathématiques et la vie réelle, entre différentes branches des mathématiques et entre les mathématiques et les autres disciplines (Bouchamma, Clavette et Levesque, 2007). Ces principes veulent favoriser le développement de la culture mathématique chez tous les élèves dans le sens de PISA (Schleicher, 1999). Ils s'inscrivent également dans une perspective socioconstructiviste, tout autant qu'ils reflètent le courant didactique associé aux standards américains (NCTM, 2000) et à la réforme de l'enseignement des mathématiques au Québec (Ministère de l'Éducation du Québec, 2000) et dans d'autres pays francophones. Ce type de problèmes contribuerait éventuellement à la construction de démarches plus créatives selon Cline (1999), Sheffield (2003), Freiman (2006), ainsi que Freiman et Sriraman (2007).

Par contre, nos résultats révèlent aussi un faible pourcentage de problèmes répondant aux autres critères de richesse, soit d'amener l'élève à justifier ses choix, à poser d'autres problèmes, à permettre la découverte de régularités, de généralisations, de preuves, ou d'être mal défini (données superflues et manquantes). II semble donc y avoir un besoin d'enrichir le cadre conceptuel des problèmes posés dans la CASMI en y intégrant les types de tâches riches sous-utilisées jusqu'à maintenant et d'en étudier plus en profondeur le potentiel pour développer la créativité dans les solutions proposées par les élèves. Si l'on accepte les conclusions de Corbeil, Pelletier et Pallascio (2001) à l'effet que les tâches complexes intégrant ces critères permettent à l'élève d'aller au-delà d'une application des règles ou des concepts mathématiques et d'entamer un processus plus créatif de découverte des régularités, de questionnement, de généralisation et de preuve, il conviendrait de poser davantage de problèmes de ce genre dans la CASMI.

Quant à la créativité mathématique, on retrouve ses composantes dans les espaces virtuels collectifs de solutions dans près de la moitié des problèmes posés dans la CASMI. Ce niveau modeste de créativité soulève plusieurs questions. Les enseignants entraînent-ils leurs élèves en leur montrant la «bonne stratégie » et la «bonne façon » d'arriver à la seule réponse possible? Les concepteurs du site doivent-ils enrichir les problèmes, les rendre plus ouverts, poser plus de questions menant à l'investigation de différentes possibilités? Peut-on développer chez l'élève une culture de questionnement de haut niveau lui permettant d'exercer sa pensée critique et de modifier un problème ou en poser un autre plus riche, même si le problème de départ est en apparence banal et suggère implicitement une démarche apprise? Bien sûr, notre recherche ne fournit pas de pistes claires quant aux moyens de susciter plus de créativité dans les solutions aux problèmes posés dans la CASMI. Cependant, des auteurs comme Sheffield (2009) avancent qu'un travail sur la compréhension plus profonde des concepts et des propriétés mathématiques est nécessaire et que celui-ci gagne à se faire sous forme de partage et de discussion entre les élèves. Cette forme de collaboration est non seulement possible en salle de classe, mais aussi dans une communauté 
virtuelle comme la CASMI. En effet, des formes de communication virtuelle, telles que le forum de discussion, pourraient être examinées pour favoriser la résolution créative de problèmes par un groupe d'élèves de façon collaborative (Freiman et Michaud, 2009; Kuntz, 2005; LeBlanc, René de Cotret et Freiman, 2010; Stahl, 2009). On sait par ailleurs que le forum de discussion est rarement utilisé spontanément par les élèves dans la CASMI (Freiman et Lirette-Pitre, 2009). En fait, les élèves utilisent cet outil principalement lorsqu'une activité d'apprentissage exige des échanges en ligne. En posant des problèmes qui exigent une résolution en collaboration, les responsables de la CASMI favoriseraient l'exploitation du forum de discussion, donnant ainsi accès à un environnement riche en informations et ce, autant pour l'enseignant que pour l'élève. Dans le forum de discussion, des interactions écrites, qui ne correspondent pas nécessairement aux résultats attendus, sont conservées. Dès lors, le forum s'avère un outil intéressant pour la conceptualisation, car il présente des informations qui, en plus de permettre à l'enseignant de mieux comprendre le raisonnement des élèves, permettent aussi aux élèves de mieux comprendre leur propre raisonnement. Des recherches dans ce domaine ont déjà été menées par les responsables du site Math Forum (Stahl, 2009). Dans ces recherches, les élèves regroupés en " équipes mathématiques virtuelles » résolvent des problèmes en ligne et ont l'occasion de donner un sens aux concepts mathématiques qui y sont reliés. Davantage de recherches sur cette pratique demandent toutefois à être réalisées.

Enfin, malgré le fait que la créativité observée dans les solutions aux problèmes considérés dans cette étude s'avère relativement restreinte, les résultats obtenus confirment l'existence d'un lien entre la richesse des problèmes mathématiques posés dans le site de la CASMI et la créativité mathématique de l'espace virtuel collectif de solutions, plus précisément sur les plans de la fluence et de l'originalité. Ce constat va dans le même sens que les suggestions d'auteurs tels que Cline (1999), Sheffield (2003), Freiman (2006) et Freiman et Sriraman (2007), qui avancent que les problèmes plus riches ont possiblement le potentiel d'apporter des solutions plus créatives. Ce résultat milite donc en faveur d'un effort accru pour augmenter la richesse des problèmes posés dans la CASMI. Un tel effort semble d'autant plus nécessaire que, comme nous l'avons noté précédemment, cette communauté virtuelle est l'une des rares ressources conçues dans et pour un milieu minoritaire francophone. On peut donc faire l'hypothèse que l'accroissement de la richesse mathématique des problèmes posés dans la CASMI pourrait avoir des retombées positives sur les apprentissages et la créativité des élèves vivant dans un tel milieu.

Enfin, il convient de se questionner sur les limites de cette recherche, particulièrement en ce qui a trait aux définitions de certains critères de la richesse des problèmes mathématiques et de la créativité. Rappelons que les définitions et les critères adoptés pour construire les grilles d'évaluation de la richesse des problèmes et de la créativité des solutions utilisées dans cette étude constituent des choix en accord avec notre positionnement épistémologique quantitatif qui appelle le recours à des critères observables et mesurables, déjà reconnus dans les écrits scientifiques du domaine. II demeure cependant que la richesse et la créativité mathématiques constituent des construits polysémiques dont l'étude peut être abordée sous plusieurs angles et de plusieurs points de vue différents. D'autres définitions et d'autres critères sont certainement possibles. Entre autres, Piggot (2007) mentionne comme critère de richesse d'un problème son potentiel d'enrichir les habiletés mathématiques des élèves ainsi que de leur faire découvrir de nouveaux concepts mathématiques. Un tel critère pourrait constituer autant un enrichissement potentiel d'une grille d'évaluation quantitative qu'une problématique d'investigation qualitative en soi. Bien sûr, cette 
recherche se voulait exploratoire et aucune conclusion définitive ne peut être tirée par rapport à la richesse des problèmes posés dans la CASMI, ni sur la créativité des solutions proposées par ses membres. Par contre, cette recherche jette un regard sur un domaine d'investigation encore trop peu exploré. Son intérêt se situe moins dans les réponses provisoires qu'elle apporte que dans les multiples questions qu'elle soulève.

\section{Conclusion}

Cette recherche met en évidence que les problèmes mathématiques posés dans le site de la CASMI sont en général modestes sur le plan de la richesse, selon les critères que nous avons considérés, et qu'il y a des traces de créativité dans les espaces virtuels collectifs de solutions. Cette recherche permet aussi d'observer un lien entre la richesse d'un problème mathématique et la créativité mathématique exprimée dans son espace virtuel collectif de solutions en termes de fluence et d'originalité. Ces constats, associés au fait que la CASMI est souvent la seule ressource en français disponible aux jeunes néo-brunswickois, pointent vers un besoin d'augmenter les efforts pour offrir aux jeunes des milieux minoritaires francophones davantage de possibilités de solutionner des problèmes plus riches afin d'augmenter leur créativité et de renforcer leurs apprentissages en mathématiques.

Malgré ses limites, la présente étude montre l'intérêt de poursuivre des recherches sur l'adéquation entre les ressources disponibles et les objectifs de formation en regard de la créativité mathématique. Deux axes de recherches peuvent donc être envisagés. D'une part, il conviendrait d'effectuer des recherches plus approfondies sur les critères à considérer dans l'élaboration de tâches riches en mathématiques en lien avec le développement de la créativité dans ce domaine. D'autre part, les facteurs qui contribuent à augmenter l'efficacité de ressources telles que les communautés virtuelles d'apprentissages, comparativement aux moyens traditionnels tels que les manuels scolaires, en ce qui a trait au développement de la créativité mathématique, demandent à être explorés davantage. De telles recherches permettraient à la fois de renforcer les balises théoriques et de planifier les stratégies à mettre en œuvre dans ce domaine.

\section{Références}

Bouchama, Y., Clavette, F. et Levesque, P. (2007). Porte d'entrée dans la réussite en mathématiques. Moncton : Éditions de la Francophonie.

Chan, C. M. E. (2008). The use of mathematical modeling tasks to develop creativity. Paper presented at the $11^{\text {th }}$ International Congress on Mathematical Education, July 6-13, Monterrey, Mexico.

Cline, S. (1999). Giftedness Has Many Faces : Multiple Talents and Abilities in the Classroom. Delray Beach : Winslow Press; The Foundation for Concepts in Education.

Corbeil, N., Pelletier, M. et Pallascio, R. (2001). Les situations-problèmes : au cœur de la réforme en mathématiques. Instantanés mathématiques, 32(3), 14-27.

Diezmann, C. et Watters, J. (2004). Challenge and connectedness in the mathematics classroom; using lateral strategies with gifted elementary students. Paper presented at the $10^{\text {th }}$ International Congress on Mathematical Education, July 4-11, Copenhagen, Denmark.

Field, A. (2005). Discovering Statistics Using SPSS (2nd Edition). London : Sage.

Freiman, V. (2006). Problems to discover and to boost mathematical talent in early grades : a challenging situations approach. The Montana Mathematics Enthusiast, 3(1), 51-75.

Freiman, V. et Lirette-Pitre, N. (2009). Building a virtual learning community of problem solvers : example of CASMI community. ZDN, 42(1-2), 245-256.

Freiman, V., Lirette-Pitre, N. et Manuel, D. (2007). Building a virtual learning community of problem solvers : example of CASMI Community. Paper presented at the Second International Symposium on Mathematics and its Connections to the Arts and Sciences, University of Southern Denmark, Odense, Denmark. 
Freiman, V. et Manuel, D. (2007). Apprentissage des mathématiques. Dans S. Blain, J. Beauchamp, C. Essiembre, V. Freiman, N. Lirette-Pitre, D. Villeneuve, H. Fournier, P. Clavet, M. Cormier et D. Manuel (Dir.), Les effets de I'utilisation des ordinateurs portatifs individuels sur l'apprentissage et les pratiques d'enseignement (p. 232-283). Moncton : Centre de recherche et de développement en éducation, Université de Moncton.

Freiman, V., Manuel, D. et Lirette-Pitre, N. (2007). CASMI virtual learning collaborative environment for mathematical enrichment. Understanding our Gifted, Summer 2007, 20-23.

Freiman, V. et Michaud, D. (2009). One mathematical formula in the science textbook : looking into innovative potential of interdisciplinary mathematics teaching. Dans A. Rogerson (Dir.) Proceedings of the Tenth International Conference : Models in Developing Mathematics Education (p. 184-188). Dresden : Hochschule für Technik und Wirtschaft.

Freiman, V. et Sriraman, B. (2007). Does mathematics gifted education need a philosophy of creativity? Mediterranean Journal for Research in Mathematics Education, 6(1 et 2), 23-46.

Greenes, C. (1997). Honing the abilities of the mathematically promising. Mathematics Teacher, 90(7), 582-586.

Hancock, C. L. (1995). Implementing the assessment standards for school mathematics : Enhancing mathematics learning with open-ended questions. Mathematics Teacher, 88(6), 496-499.

Hashimoto, Y. (1997). The methods of fostering creativity through mathematical problem solving. International Reviews on Mathematical Education, 29(3), 86-87.

Haylock, D. (1997). Recognizing mathematical creativity in school children. International Reviews on Mathematical Education, 29(3), 68-74.

Haylock, D. W. (1987). A framework for assessing mathematical creativity in school children. Education Studies in Mathematics, 18(1), 59-74.

Hershkovitz, S. Peled, I. et Littler, G. (2009). Mathematical creativity and giftedness in elementary school : Task and teacher promoting creativity for all. Dans R. Leikin, A. Berman et B. Koichu (Dir.), Creativity in Mathematics and the Education of Gifted Students (p. 255-270). Rotterdam : Sense Publisher.

Jean, C.-É. (2001). Récréomath. < http://www.recreomath.qc.ca/lex.htm >.

Kitchner, K. S. (1983). Cognition, metacognition, and epistemic cognition : A three-level model of cognitive processing. Human Development, 26, 222-232.

Klavir, R. et Hershkovitz, S. (2008). Teaching and evaluating "Open-Ended" Problems [Electronic Version]. International Journal for Mathematics Teaching and Learning. May $20^{\text {th }}$. < http://www.cimt.plymouth.ac.uk/journal/klavir.pdf $>$

Knighton, T., Brochu, P. et Gluszynski, T. (2010). À la hauteur : Résultats canadiens de l'étude PISA de l'OCDE. La performance des jeunes canadiens en lecture, en mathématiques et en sciences. Premiers résultats de 2009 pour les canadiens de 15 ans. Ottawa : Conseil des ministres de l'éducation; Statistiques Canada.

Krutetskii, V. A. (1976). The Psychology of Mathematical Abilities in School Children. Chicago : University of Chicago Press.

Kuntz, G. (2005). Résolution collaborative de problèmes ouverts : un problème babylonien. Bulletin de l'APMEP, 456, $123-131$.

Langlais, M., Vézina, N. et Freiman, V. (2005). Le Chantier d'Apprentissages Mathématiques Interactifs (CAMI) accompagne la réforme au Nouveau-Brunswick: Mathématique virtuelle à l'attention du primaire. < http://spip.cslaval.qc.ca/mathvip/rubrique.php3?id_rubrique=18 >

LeBlanc, M., René de Cotret, S. et Freiman, V. (2010). Contre-exemple ou exception? Là est la question. Étude de l'évolution d'une discussion en ligne. Présentation faite au colloque du Groupe de didacticiens des mathématiques (GDM), 10-12 juin. Moncton, Nouveau-Brunswick.

Leikin, R. (2007). Habits of mind associated with advanced mathematical thinking and solution spaces of mathematical tasks. Paper presented at The Fifth Conference of the European Society for Research in Mathematics Education (CERME-5) (2226 Feb.) Larnaca, Cyprus.

Lester, F. K. et Kehle, P. E. (2003). From problem solving to modelling: The evolution about ressearch on complex mathematical activity. Dans R. A. Lesh et H. M. Doerr (Dir.), Beyond Constructivism : Models and Modeling Perspectives on Mathematics Problem Solving, Learning and Teaching. Mahwah : Lawrence Erlbaum.

Mann, E. (2005). Mathematical creativity and school mathematics : Indicators of mathematical creativity in middle school students. Thèse de doctorat inédite. Thorss : University of Connecticut.

Mann, E. (2006). Creativity : The essence of mathematics. Journal for the Education of the Gifted, 30(2), 236-260.

Meissner, H. (2005). Creativity and mathematics education. Paper presented at the The $3^{\text {rd }}$ East Asia Regional Conference on Mathematics Education, August 7-12. Shanghai, China. < http://www.math.ecnu.edu.cn/earcome3/sym1/sym104.pdf >

Ministère de l'Éducation et du Développement de la Petite Enfance du Nouveau-Brunswick (2011). Programmes d'études : Mathématiques 30131. Fredericton : Ministère de l'Éducation; Direction des services pédagogiques.

Ministère de l'Éducation du Québec (2000). Programme de formation de l'école québécoise : Éducation préscolaire, enseignement primaire. Québec : Ministère de l'Éducation du Québec. 
Murphy, E. (2004). Identifying and measuring ill-structured problem formulation and resolution in online asynchronous discussions. Canadian Journal of Learning and Technology, 30(1), 5-20.

NCTM (2000). Principles and Standards for School Mathematics. Reston : National Council of Teachers of Mathematics.

Pallascio, R. (2005). Les situations-problèmes : un concept central du nouveau programme de mathématique. Vie Pédagogique, 136, 32-35.

Petrowski, M. J. (2000). Creativity research : implications for teaching, learning and thinking. Reference Services Review, 28(4), 204-214.

Piggott, J. (2007). Cultivating creativity. Mathematics Teaching Incorporating Micromath, 202, 3-6.

Poirier, L. (2001). Enseigner les maths au primaire. Québec : Éditions du renouveau pédagogique.

Poirier L. (2010). La formation des enseignants en milieu minoritaire au Nouveau-Brunswick : quelques pistes de réflexion. Dans J. Proulx et L. Gattuson (Dir.) Formation des enseignants en mathématiques : tendances et perspectives actuelles, $\mathrm{p}$. 225-231. Sherbrooke : Éditions du CRP.

Renninger, K. A. et Shumar, W. (2004). The centrality of culture and community to participant learning at and with the Math Forum. Dans S. Barab, R. Kling et J. Gray (Dir.), Designing for Virtual Communities in the Service of Learning. New York: Cambridge University Press.

Robinson, K. (2009). Why creativity now? A conversation with Sir Ken Robinson. Educational Leadership (September 2009), 22-23.

Runco, M. A. (1993). Divergent thinking, creativity and giftedness. Gifted child Quarterly, 37(1), 16-22.

Schleicher, A. (1999). Measuring Student Knowledge and Skills : A New Framework for Assessment. Paris : Organization for Economic Co-operation and Development.

Sheffield, L. (2003). Extending the Challenge in Mathematics. Newbury Park : TAGT \& Corwin Press.

Sheffield, L. (2008). Promoting creativity for all students in mathematics education : An overview. Paper presented at the $11^{\text {th }}$ International Congress on Mathematical Education (July 6-13). Monterrey, Mexico.

Sheffield, L. (2009). Developing mathematical creativity - Questions may be the answer. Dans R. Leikin, A. Berman et B. Koichu (Dir.), Creativity in Mathematics and the Education of Gifted Students (p. 87-100). Rotterdam : Sense Publishers.

Singh, B. (1988). Teaching-Learning Strategies and Mathematical Creativity. Delhi : Mittal Publications.

Sriraman, B. (2004). The characteristics of mathematical creativity. The Mathematics Educator, 14(1), 19-34.

Stahl, G. (2009). Studying Virtual Math Teams. New-York : Springer.

Tabachnick, B. G. et Fidell, L. S. (2007). Using Multivariate Statistics (5th ed.). Boston : Allyn and Bacon.

Takahashi, A. (2000). Open-ended Problem Solving Enriched by the Internet. NCTM annual meeting. < http://www.mste.uiuc.edu/users/aki/open_ended/>

Treffinger, D. J., Young, G. C., Selby, E. C. et Shepardson, C. (2002). Assessing Creativity : A Guide for Educators. Storrs : University of Connecticut, The National Research Center on the Gifted and Talented.

Van der Maren, J.-M. (1996). Méthodes de recherche pour l'éducation ( $2^{e}$ éd.). Montréal : Presses de l'Université de Montréal.

Zambo, D. et Brozo, W. G. (2008). Bright Beginnings for boys. Engaging Young Boys in Active Literacy. Newark : International Reading Association. 


\section{Annexe A}

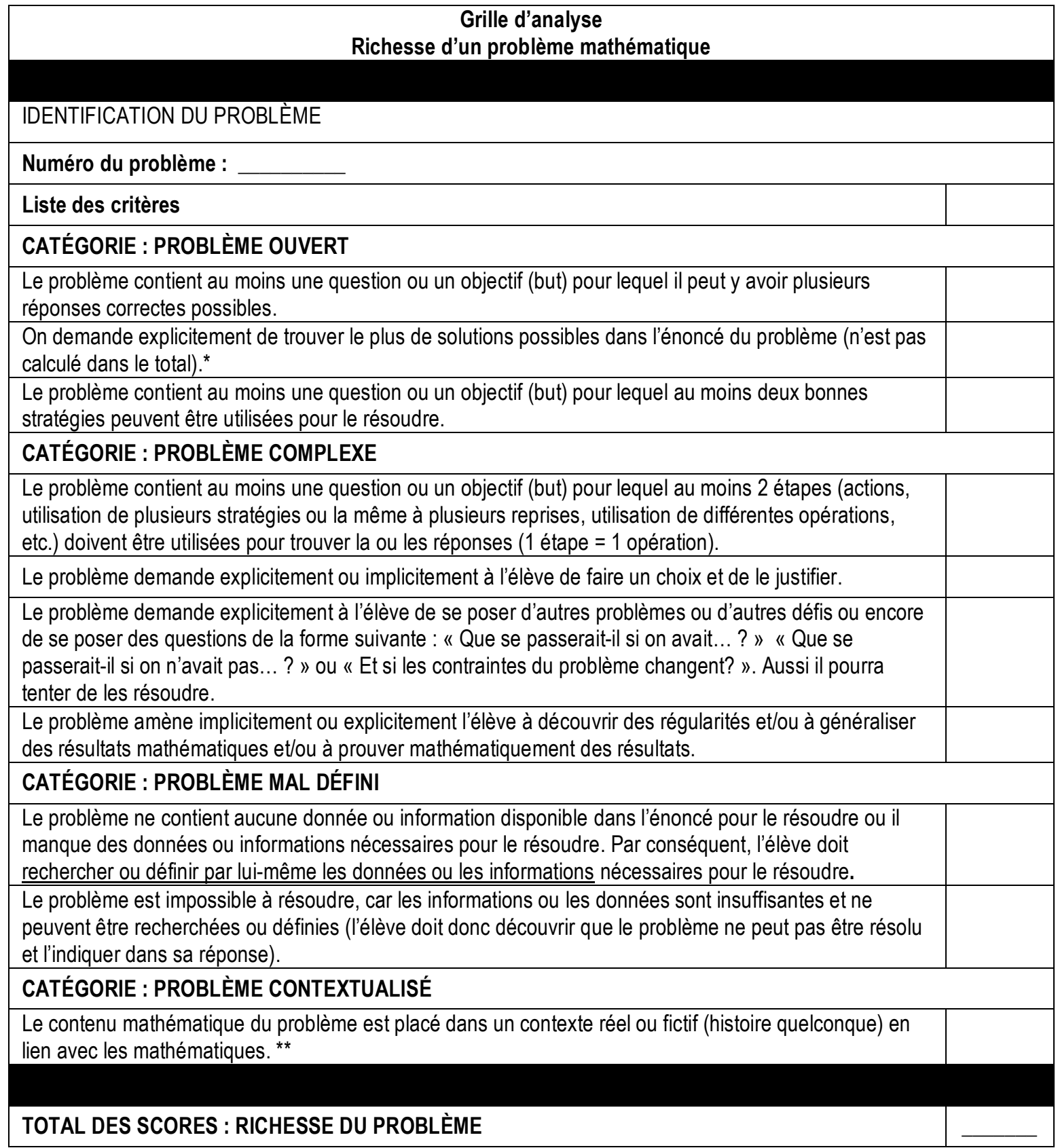

${ }^{*}$ Ce critère sert surtout à faire la comparaison entre les problèmes demandant explicitement de trouver le plus de solutions possibles et ceux qui ne le demandent pas. C'est pourquoi nous ne calculons pas ce résultat dans le score de la richesse du problème puisqu'il est inclu dans le critère précédent.

**Nous ne considérons pas une situation dans laquelle une personne résout un problème dans un manuel scolaire ou un livre d'énigmes comme une situation contextuelle. Nous considérons ces cas comme étant des contextes purement mathématiques. 


\section{Annexe B}

Grille d'analyse de la créativité mathématique de l'espace virtuel collectif des solutions à un problème posé dans le site de la CASMI

\begin{tabular}{|c|c|}
\hline Composante & \\
\hline \multirow[b]{2}{*}{ 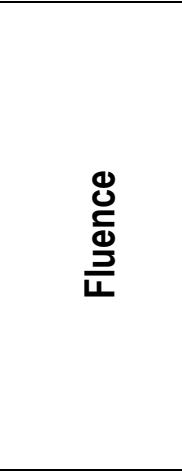 } & $\begin{array}{l}\text { Attribution du score: } \\
1 \text { point pour chaque bonne réponse ou nouveau problème correct formulé dans l'espace virtuel } \\
\text { collectif de solutions. }\end{array}$ \\
\hline & $\begin{array}{l}\text { Exceptions : } \\
\text { - L'utilisation d'éléments neutres (ex. } 0 \text { pour l'addition ou } 1 \text { pour la multiplication) ne } \\
\text { contribue pas à augmenter le nombre de bonnes réponses. } \\
\text { - Les fractions équivalentes (ex. } \frac{1}{2} \text { et } \frac{3}{6} \text { comptent comme une seule et même réponse. } \\
\text { - Si la règle mathématique demande explicitement de considérer plus d'une réponse } \\
\text { (comme avec les équations quadratiques ou les valeurs absolues), ces réponses sont } \\
\text { comptées comme une seule. }\end{array}$ \\
\hline \multirow{2}{*}{ 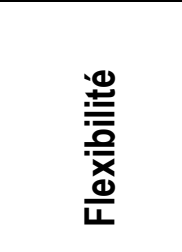 } & $\begin{array}{l}\text { Attribution du score : } \\
1 \text { point pour chaque stratégie correcte utilisée pour résoudre le problème dans l'espace virtuel } \\
\text { collectif de solutions. }\end{array}$ \\
\hline & Nous nous basons sur la liste des stratégies élaborées par Jean (2001) \\
\hline \multirow[b]{2}{*}{ 黑 } & $\begin{array}{l}\text { Attribution du score : } \\
\text { On utilise la formule suivante : } O=\frac{(2 \times T O)+(M O)}{S O L} \text { où O représente le score d'originalité, } \\
\text { TO représente le nombre de solutions très originales, } \mathrm{MO} \text { le nombre de solutions moyennement } \\
\text { originales et } \mathrm{SOL} \text { signifie le nombre total de solutions dans l'espace virtuel collectif de solutions. }\end{array}$ \\
\hline & $\begin{array}{l}\text { Définitions : } \\
\text { - TO représente le nombre de solutions qui ont une réponse et/ou une stratégie utilisée } \\
\text { par } 5 \% \text { ou moins des participants dans l'espace virtuel collectif de solutions (plus haut } \\
\text { niveau). } \\
\text { MO représente le nombre de solutions qui ont une réponse et/ou une stratégie utilisée } \\
\text { par entre } 5,01 \% \text { et } 20 \% \text { des participants dans l'espace virtuel collectif de solutions } \\
\text { (deuxième plus haut niveau). } \\
\text { - Si une solution contient un élément de chaque niveau (ex. : réponse très originale (TO) } \\
\text { et stratégie moyennement originale (MO), nous classifions la solution au plus haut } \\
\text { niveau. (Dans l'exemple entre parenthèses au début de cette énumération, cette solution } \\
\text { serait très originale). }\end{array}$ \\
\hline
\end{tabular}

